\title{
Recommendations for the management of non-alcoholic fatty liver disease (NAFLD)
}

\author{
Recommendations of the Polish Group of Experts for Non-Alcoholic Fatty Liver Disease (PGE-NAFLD): \\ Krzysztof Tomasiewicz, Robert Flisiak, Waldemar Halota, Jerzy Jaroszewicz, Dariusz Lebensztejn, Wojciech Lisik, \\ Piotr Małkowski, Małgorzata Pawłowska, Anna Piekarska, Krzysztof Simon, Olga Tronina
}

The Polish Group of Experts for Non-Alcoholic Fatty Liver Disease (PGE-NAFLD) is a multidisciplinary team of hepatology experts established by the Polish Association for the Study of Liver and the Polish Society of Epidemiology and Infectious Diseases.

The recommendations for the management of non-alcoholic fatty liver disease have been developed by a group of experts in hepatology under the auspices of the Polish Association for the Study of Liver and the Polish Society of Epidemiology and Infectious Diseases. The recommendations are intended to help physicians understand the most current knowledge of NAFLD and apply it in clinical practice. Depending on the clinical situation, it may be necessary to individualize the management.

\section{Definition and epidemiology}

Non-alcoholic fatty liver disease (NAFLD) is a growing clinical and epidemiological problem worldwide. The prevalence of the disease is correlated with the level of development of a given society, however increasingly across the world the problem affects various age and social groups $[1,2]$. The definition of NAFLD should include both different stages of the disease and the wide spectrum of clinical manifestations involving not only the liver. Most patients with NAFLD experience nutritional and metabolic disorders, mainly obesity, diabetes and dyslipidaemia. The definition of NAFLD requires that there is evidence of hepatic steatosis by imaging and/or histopathological examination (preferred option) and there are no other causes for hepatic fat accumulation, primarily excessive alcohol consumption, long-term use of medications inducing hepatic steatosis, infection by steatogenic pathogens (e.g. genotype 3 of the hepatitis $\mathrm{C}$ virus) and hereditary lipid disorders $[3,4]$.

Histologically, NAFLD may be further categorized into non-alcoholic fatty liver (NAFL) and non-alcoholic steatohepatitis (NASH). The distinction between stages of NAFLD is of fundamental importance for prognosis and therapy. NAFL is defined as the presence of $>5 \%$ of steatotic hepatocytes without features of hepatocyte injury and ballooning degeneration. The diagnosis of NASH requires the presence of inflammation and hepatocyte injury (most commonly ballooning degeneration), with fibrosis not being a prerequisite for diagnosing NASH [4-6].

The prevalence of NASH in the general population and across different regions/countries is difficult to es- timate. Since NASH must be confirmed histopathologically, only in some patients the diagnosis can be made in a methodologically correct manner. On the other hand, the obesity "epidemic", growing prevalence of diabetes and evidence of hepatic steatosis in imaging studies in a large number of patients, give grounds to assume that NAFLD, but also NASH, affect a significant percentage of the population $[7,8]$. One of the largest meta-analyses, published by Younossi et al., shows that NASH confirmed by liver biopsy affects almost $60 \%$ of patients with liver disease (biopsy for clinical indications) and between 2 and $6 \%$ of the general population [9]. If these estimates are correct, NASH and its consequences occur much more commonly than any other liver disease.

\section{Risk factors}

Recognized factors increasing the risk of NAFLD and/or associated with the development of the disease include [10-12]:

1. High body mass index (BMI) [linear correlation with risk escalation $>30 \mathrm{~kg} / \mathrm{m}^{2}$ ] and abdominal obesity;

2. Type 2 diabetes;

3. Dyslipidaemia - high level of triglycerides (TG) in blood serum (depending on the sex and ethnicity, but the recognized threshold value is $150 \mathrm{mg} / \mathrm{dl}$, low level of high-density lipoproteins (HDL) $(<40 \mathrm{mg} / \mathrm{dl}$ in men and $<50 \mathrm{mg} / \mathrm{dl}$ in women);

4. Age - the prevalence of NAFLD rises with age;

5. Male sex - the prevalence of NAFLD is twice as high in men as in women;

6. Long-term consumption of even moderate amounts of alcohol in combination with excessive caloric intake and/or obesity. 
The role of the ethnicity factor is attributed to genetic predisposition, namely variation in the PNPLA3 gene. The presence of the PNPLA3 I148M and TM6SF2 E167K variants may elevate the risk of development of NAFLD/NASH, and symptoms of the disease may be unaccompanied by features of the metabolic syndrome [13]. In cases where genetic testing for these variants is available, their presence can be assessed in selected clinical situations, however genetic tests are not recommended routinely [14].

\section{Diagnosis of NAFLD/NASH}

Cost efficiency analyses available in the literature have shown that widespread screening for NAFLD should not be recommended. However, caution should be exercised in patients with type 2 diabetes and suspicion of NASH, especially when advanced liver disease is suspected $[15,16]$. The unambiguous diagnosis of NASH/NAFLD can pose difficulties.

The diagnosis of NAFLD requires: (1) evidence of hepatic steatosis found by imaging (non-invasive methods) or histopathologial examination (liver biopsy); (2) no alcohol intake or moderate alcohol consumption; (3) exclusion of other causes of hepatic steatosis; (4) exclusion of other causes of liver disease [3, 6]. In addition to NAFLD, the most common causes of hepatic steatosis include alcohol abuse, HCV infection, drugs (especially corticoids, tetracyclines, oestrogens), parenteral nutrition, Wilson's disease and malnutrition. Furthermore, the differential diagnosis should include haemochromatosis, autoimmune liver diseases and alpha-1-antitrypsin deficiency. NAFLD can coexist with other nosological entities. Extensive differential diagnostics is of particular significance in the presence of advanced liver fibrosis $[2,14]$. Appropriate diagnostic work-up is also important in each case of suspected NASH.

The presence of hepatic steatosis can be diagnosed by non-invasive and invasive methods:

\section{Non-invasive methods}

A. Ultrasonography - basic method, widely available and inexpensive; enables assessment of liver structure also with respect to focal lesions or biliary pathologies. Ultrasonography does not usually reliably detect hepatic steatosis when less than $20 \%$ hepatocytes are affected. Furthermore, the results may be unreliable in patients with BMI $>40 \mathrm{~kg} / \mathrm{m}^{2}$ or inadequately prepared for the examination.

B. Computed tomography - slightly superior to ultrasonography with regard to the assessment of hepatic steatosis, however more expensive and less widely available than the latter method.
C. Magnetic resonance imaging - recognized as the "gold standard" among imaging techniques; enables visualization of even mild steatosis. It is the basic non-invasive method which can be used to assess response to therapy [17]. Disadvantages include high cost and long duration of the examination.

D. Magnetic resonance spectroscopy - a method with similar advantages and disadvantages as MRI. From the technical point of view, it involves a separate analysis of fat and water signals, enabling quantification of fat content in the liver. From the practical perspective, on account of less extensive experience, limited availability and higher cost, magnetic resonance spectroscopy is considered to be less useful as a diagnostic modality in NAFLD/ NASH than MRI $[17,18]$.

E. Elastography with CAP (controlled attenuation parameter) option - because of insufficient data and lack of reliable studies comparing the usefulness of this tool with the above-mentioned imaging methods CAP cannot, as yet, be recommended in the diagnostic work-up for NAFLD/NASH $[19,20]$.

F. Serum biomarkers - there are multiple diagnostic tools based on the analysis of serum concentrations of different proteins or substances. Their usefulness in assessing the stage of fibrosis is viewed rather critically in relation to elastographic methods. Tests that have been validated for NAFLD include NAFLD fibrosis score (NFS) and FIB-4.

The suitability of biomarkers for assessing steatosis and/or determining the presence of NASH is the subject of intensive research. One of the most promising tests is based on measuring cytokeratin-18 (CK-18) fragments generated during cell death (M65) or apoptosis (M30). Because of its relatively low sensitivity (66\%) and specificity ( $82 \%)$, and more difficult accessibility of CK-18 measurements, the method is currently not recommended for the diagnosis of NASH [21].

2. Invasive methods (liver biopsy)

Despite its widely known limitations, histological evaluation of material obtained by liver biopsy is the only method that reliably differentiates NAFL from NASH. Bioptate analysis is the only method to identify features typical of NASH: coexisting steatosis, lobular inflammation and balloon degeneration. Other features that can be seen in NASH, but are not necessary for the diagnosis include portal inflammation, polymorphonuclear infiltrates, Mallory-Denk bodies, apoptotic bodies or perisinusoidal fibrosis. Scoring systems for assessing disease stage are also used, of which the most important are NAS (NAFLD Activity Score) and SAF (steatosis, activity and fibrosis) [4, 22]. 
The decision to perform a biopsy should be made based on the patient's complete clinical picture and the presence of risk factors for NASH development, especially in cases with no defined aetiology of liver disease.

\section{Therapeutic management}

Pharmacological treatment of liver disease should be reserved for patients diagnosed with NASH, and with significant steatosis and advanced liver disease. Extensive differential diagnostics is necessary to determine the cause of fibrosis.

Since NAFLD is accompanied by systemic health conditions, multifaceted management by multidisciplinary teams is required. Testing for diabetes and, if needed, pharmacotherapy is required in all patients with NAFLD. Hepatic steatosis without inflammation also requires therapeutic management, with good results achieved by lifestyle modification and initiating treatment of concomitant diseases. Patients with NAFLD require oncological alertness because of recent reports of increased incidence of cancer, including primary hepatocellular carcinoma (HCC), also in individuals without cirrhosis [23].

1. Lifestyle modification - an appropriate diet, increased physical activity and weight loss are the first line of intervention in patients with NAFLD/NASH. Body weight reduction by $>5 \%$ has been shown to decrease hepatic steatosis, and by $>10 \%$ to contribute to histological improvement in patients with NASH $[24,25]$. This management also helps to reduce the risk of cardiovascular diseases.

An increased level of physical activity is known to induce body weight reduction, but the effect of exercise on improving the histological picture has not been demonstrated unequivocally. Physical activity must be combined with diet [26]. Basic dietary recommendations include lowering the calorie content of meals (decrease in daily caloric intake by $500-1000$ kcal) and avoidance of processed foods, products and drinks that are high in fructose.

2. Insulin sensitizers

A. Metformin - despite some studies demonstrating a positive effect of metformin on the activity of liver enzymes and a decrease in insulin resistance, the drug has not been shown to affect the course of NASH and the histological picture [27, 28]. Consequently, metformin is not recommended for the treatment of NASH.

B. Thiazolidinediones - the latest research shows that pioglitazone produces a beneficial effect in patients with NASH both with and without diabetes. However, increased insulin sensitivity and reduced hepatic fibrosis apply to a greater extent to diabetic patients [29-31]. Such treatment may be considered on a case-by-case basis, however, the therapy will perhaps be recommended after conducting further studies.

3. Vitamin $\mathrm{E}$ - the benefit of vitamin $\mathrm{E}$ in the treatment of NASH is based on its antioxidant activity. Results of randomized trials indicate that vitamin $\mathrm{E}$ at a dose of $800 \mathrm{mg} / \mathrm{d}$ contributes to the normalization of aminotransferase activity, reduction in steatosis and inflammation, and even balloon degeneration in patients with NASH without diabetes, however without any effect on fibrosis $[32,33]$. There are concerns about the long-term effect of vitamin $\mathrm{E}$ on prostate cancer in men over 50 years of age [34]. Further studies are, however, necessary.

4. Ursodeoxycholic acid (UDCA) - study results are divergent. Observations have been conducted for different doses ranging from 10 to $35 \mathrm{mg} / \mathrm{kg}$. UDCA is believed to have a potentially positive effect on biochemical activity, particularly in combination with, for example, vitamin E $[35,36]$. UDCA products are not indicated for the treatment of NASH in the USA. According to the proposed management algorithm the efficacy should be verified by assessing the activity of aminotransferases after 3-4 months; a decrease in baseline values by at least $1 / 3$ justifies the continuation of treatment.

5. Agonists of FXR (farnesoid X receptor) - in preclinical studies they show a number of benefits in NAFLD/ NASH due to their metabolic activity causing stabilization of lipid and carbohydrate metabolism, and their immunomodulatory and anti-inflammatory effects. Clinical trials are underway to investigate their natural ligands (CA and CDCA), semi-synthetic modified bile acids (obeticholic acid, OCA) and semi-synthetic non-steroidal molecules (GW4064 and WAY-362450). Preliminary findings suggest that these medications may reduce steatosis and inflammation in NAFLD/ NASH [37]. However, their effect depends on the duration of treatment and the risk of adverse reactions requiring discontinuation of therapy (e.g. pruritus).

6. Agonists of peroxisome proliferator-activated receptors (PPARa) - Clinical studies on fibrates have demonstrated their beneficial effects in dyslipidaemia accompanying NASH. Fenofibrate treatment in patients with biopsy-confirmed NASH has led to a reduction in the number of patients with elevated aminotransferase (ALT, AST) and gamma-glutamyltranspeptidase (GGT) activity, and baloon degeneration evaluated by biopsy. However, there have been no significant changes in terms of steatosis, inflammation and fibrosis. Short-term treatment with 
bezafibrate (2-8 weeks), in combination with appropriate diet and increased physical activity, has been found to reduce microvesicular steatosis. Short-term 4-week treatment of NASH with gemfibrozil has led to a decrease in AST and GGT activity. A year-long therapy with clofibrate has not been demonstrated to provide any benefit [38].

7. Cenicriviroc - a CCR5 co-receptor inhibitor currently undergoing the approval process. The results of clinical trials are very promising [39].

8. Statins - their use in NASH brings benefits in terms of reduction of cardiovascular risk. Long-term follow-up shows that statin therapy is safe for the liver, however there is also no clear evidence of benefits in this indication. Only isolated observations have shown histological improvement, and one of the retrospective studies has found that the incidence of hepatic cancer is $27 \%$ lower in statin-treated patients [40,41]. These data, however, are not sufficient for a definite recommendation to use statins in NASH therapy.

9. Bariatric surgery - a surgical option recommended in patients with obesity grades II or III not responding to other therapies $[42,43]$.

In recent years, there has been a significant increase in the number of clinical trials investigating new treatments for NASH. It is to be expected that the number of therapeutic options will increase in a short-term perspective, which does not diminish the importance of non-pharmacological management methods.

\section{Disclosure}

The authors report no conflict of interest.

\section{References}

1. Sayiner M, Koenig A, Henry L, et al. Epidemiology of nonalcoholic fatty liver disease and nonalcoholic steatohepatitis in the United States and the rest of the world. Clin Liver Dis 2016; 20: 205-214.

2. Chalasani N, Younossi Z, Lavine JE, et al The diagnosis and management of nonalcoholic fatty liver disease: practice guidance from the American Association for the Study of Liver Diseases. Hepatology 2018; 67: 328-357.

3. Ahmed A, Wong RJ, Harrison SA. Nonalcoholic fatty liver disease review: diagnosis, treatment, and outcomes. Clin Gastroenterol Hepatol 2015; 13: 2062-2070.

4. Yeh MM, Brunt EM. Pathological features of fatty liver disease. Gastroenterology 2014; 147: 754-764.

5. Kaswala DH, Lai M, Afdhal NH. Fibrosis assessment in nonalcoholic fatty liver disease (NAFLD) in 2016. Dig Dis Sci 2016; 61: 1356-1364.

6. Kleiner DE, Brunt EM. Nonalcoholic fatty liver disease: pathologic patterns and biopsy evaluation in clinical research. Semin Liver Dis 2012; 32: 3-13.

7. Targher G, Marchesini G, Byrne CD. Risk of type 2 diabetes in patients with non-alcoholic fatty liver disease: causal association or epiphenomenon? Diabetes Metab 2016; 42: 142-156.
8. Musso G, Gambino R, Cassader M, et al. Meta-analysis: natural history of non-alcoholic fatty liver disease (NAFLD) and diagnostic accuracy of non-invasive tests for liver disease severity. Ann Med 2011; 43: 617-649.

9. Younossi ZM, Koenig AB, Abdelatif D, et al. Global epidemiology of nonalcoholic fatty liver disease-Meta-analytic assessment of prevalence, incidence, and outcomes. Hepatology 2016; 64: 73-84.

10. Loomis KA, Kabadi S, Preii D, et al. Body Mass Index and risk of Nonalcoholic Fatty Liver Disease: two electronic health record prospective studies. J Clin Endocrinol Metab 2016; 101: 945-952.

11. Miyake T, Kumagi T, Furukawa S, et al. Non-alcoholic fatty liver disease: Factors associated with its presence and onset. J Gastroenterol Hepatol 2013; 28 (Suppl 4): 71-78.

12. Margariti E, Deutsch M, Manolakopoulos S, et al. Non-alcoholic fatty liver disease may develop in individuals with normal body mass index. Ann Gastroenterol 2012; 25: 45-51.

13. Kawaguchi T, Sumida Y, Umemura A, et al. Genetic polymorphisms of the human PNPLA3 gene are strongly associated with severity of nonalcoholic fatty liver disease in Japanese. PLoS One 2012; 7: e38322.

14. European Association for the Study of the Liver (EASL)1; European Association for the Study of Diabetes (EASD); European Association for the Study of Obesity (EASO). EASL-EASD-EASO Clinical Practice Guidelines for the management of non-alcoholic fatty liver disease. J Hepatol 2016; 64: 1388-1402.

15. Wong VW, Chalasani N. Not routine screening, but vigilance for chronic liver disease in patients with type 2 diabetes. J Hepatol 2016; 64: 1211-1213.

16. Corey KE, Klebanoff MJ, Tramontano AC, et al. Screening for nonalcoholic steatohepatitis in individuals with type 2 diabetes: a cost-effectiveness analysis. Dig Dis Sci 2016; 61: 2108-2117.

17. Kim D, Kim WR, Talwalkar JA, et al. Advanced fibrosis in nonalcoholic fatty liver disease: noninvasive assessment with MR elastography. Radiology 2013; 268: 411-419.

18. Loomba R, Wolfson T, Ang B, et al. Magnetic resonance elastography predicts advanced fibrosis in patients with nonalcoholic fatty liver disease: a prospective study. Hepatology 2014; 60: 1920-1928.

19. Vuppalanchi R, Siddiqui MS, Hallinan EK, et al. Transient elastography is feasible with high success rate for evaluation of nonalcoholic fatty liver disease (NAFLD) in a multicenter setting. Hepatology 2015; 62: 1290A.

20. Tapper EB, Challies T, Nasser I, et al. The performance of vibration controlled transient elastography in a US cohort of patients with nonalcoholic fatty liver disease. Am J Gastroenterol 2016; 111: 677-684.

21. Chen J, Zhu Y, Zheng Q, et al. Serum cytokeratin-18 in the diagnosis of non-alcoholic steatohepatitis: a meta-analysis. Hepatol Res 2014; 44: 854-862.

22. Bedossa P; FLIP Pathology Consortium. Utility and appropriateness of the fatty liver inhibition of progression (FLIP) algorithm and steatosis, activity, and fibrosis (SAF) score in the evaluation of biopsies of nonalcoholic fatty liver disease. Hepatology 2014; 60: 565-575.

23. Said A, Ghufran A. Epidemic of non-alcoholic fatty liver disease and hepatocellular carcinoma. World J Clin Oncol 2017; 8: 429-436.

24. Sung KC, Ryu S, Lee JY, et al. Effect of exercise on the development of new fatty liver and the resolution of existing fatty liver. J Hepatol 2016; 65: 791-797.

25. Vilar-Gomez E, Martinez-Perez Y, Calzadilla-Bertot L, et al. Weight loss through lifestyle modification significantly reduces 
features of nonalcoholic steatohepatitis. Gastroenterology 2015; 149: 367-378.

26. Haufe S, Engeli S, Kast P, et al. Randomized comparison of reduced fat and reduced carbohydrate hypocaloric diets on intrahepatic fat in overweight and obese human subjects. Hepatology 2011; 53: 1504-1514.

27. Li Y, Liu L, Wang B, et al. Metformin in nonalcoholic fatty liver disease: a systematic review and meta-analysis. Biomed Rep 2013; 1: 57-64.

28. Loomba R, Lutchman G, Kleiner DE, et al. Clinical trial: pilot study of metformin for the treatment of non-alcoholic steatohepatitis. Aliment Pharmacol Ther 2009; 29: 172-182.

29. Cusi K, Orsak B, Bril F, et al. Long-term pioglitazone treatment for patients with nonalcoholic steatohepatitis and prediabetes or type 2 diabetes mellitus: a randomized trial. Ann Intern Med 2016; 165: 305-315.

30. Omer Z, Cetinkalp S, Akyildiz M, et al. Efficacy of insulin-sensitizing agents in nonalcoholic fatty liver disease. Eur J Gastroenterol Hepatol 2010; 22: 18-23.

31. Aithal GP, Thomas JA, Kaye PV, et al. Randomized, placebo-controlled trial of pioglitazone in nondiabetic subjects with nonalcoholic steatohepatitis. Gastroenterology 2008; 135: 1176-1184.

32. Xu R, Tao A, Zhang S, et al. Association between vitamin $\mathrm{E}$ and non-alcoholic steatohepatitis: a meta-analysis. Int J Clin Exp Med 2015; 8: 3924-3934.

33. Dufour JF, Oneta CM, Gonvers JJ, et al. Randomized placebocontrolled trial of ursodeoxycholic acid with vitamin e in nonalcoholic steatohepatitis. Clin Gastroenterol Hepatol 2006; 4: 1537-1543.

34. Abner EL, Schmitt FA, Mendiondo MS, et al. Vitamin E and allcause mortality: a meta-analysis. Curr Aging Sci 2011; 4: 158-170.

35. Lindor KD, Kowdley KV, Heathcote EJ, et al. Ursodeoxycholic acid for treatment of nonalcoholic steatohepatitis: results of a randomized trial. Hepatology 2004; 39: 770-778.

36. Ratziu V, de Ledinghen V, Oberti F, et al. A randomized controlled trial of high-dose ursodesoxycholic acid for nonalcoholic steatohepatitis. J Hepatol 2011; 54: 1011-1019.

37. Makri E, Cholongitas E, Tziomalos K. Emerging role of obeticholic acid in the management of nonalcoholic fatty liver disease. World J Gastroenterol 2016; 22: 9039-9043.

38. Pawlak M, Lefebyre P, Staels B. Molecular mechanism of PPARa action and its impact on lipid metabolism, inflammation and fibrosis in non-alcoholic fatty liver disease. J Hepatol 2015; 62: 720-733.

39. Friedman SL, Ratziu V, Harrison SA, et al. A randomized, placebo-controlled trial of cenicriviroc for treatment of nonalcoholic steatohepatitis with fibrosis. Hepatology 2018; 67: 1754-1767.

40. Foster T, Budoff MJ, Saab S, et al. Atorvastatin and antioxidants for the treatment of nonalcoholic fatty liver disease: the St Francis Heart Study randomized clinical trial. Am J Gastroenterol 2011; 106: 71-77.

41. Chalasani N, Aljadhey H, Kesterson J, et al. Patients with elevated liver enzymes are not at higher risk for statin hepatotoxicity. Gastroenterology 2004; 126: 1287-1292.

42. Mathurin P, Hollebecque A, Arnalsteen L, et al. Prospective study of the long-term effects of bariatric surgery on liver injury in patients without advanced disease. Gastroenterology 2009; 137: 532-540.

43. Lassailly G, Caiazzo R, Buob D, et al. Bariatric surgery reduces features of nonalcoholic steatohepatitis in morbidly obese patients. Gastroenterology 2015; 149: 379-388. 